\title{
Research on Management of Doctor-Patient Risk and Status of the Perceived Behaviors of Physician Trust in the Patient in China: New Perspective of Management of Doctor-Patient Risk
}

\author{
Jiang Jie Sun $\mathbb{D}^{1,},{ }^{1,2}$ Zhi Bo Zheng, ${ }^{3}$ Xue Li Jiang, ${ }^{1}$ Wei Wei Hu, ${ }^{1}$ Jun Liu, ${ }^{4}$ Nan Zhen Ma, \\ Meng Ying Li, ${ }^{6}$ Xiao-Zhe Yan, ${ }^{7}$ Cheng sen $\mathrm{He},{ }^{6,7}$ and Li Ping Zhang $\oplus^{4,6}$ \\ ${ }^{1}$ Health Management College, Anhui Medical University, Hefei 230032, China \\ ${ }^{2}$ Anhui Higher Medical Education Cooperation Committee, Hefei 230032, China \\ ${ }^{3}$ Department of Mathematics, Baoshan University, Baoshan 678000, China \\ ${ }^{4}$ School of Humanistic Medicine, Anhui Medical University, Hefei, Anhui 230032, China \\ ${ }^{5}$ Hospital of Anhui Medical University, Hefei, Anhui, China \\ ${ }^{6}$ Clinical Medical College, Anhui Medical University, Hefei 230601, China \\ ${ }^{7}$ Department of Psychology, Anhui Medical University, Hefei, Anhui, China
}

Correspondence should be addressed to Li Ping Zhang; zhangliping@ahmu.edu.cn

Received 15 July 2020; Accepted 18 September 2020; Published 8 October 2020

Academic Editor: M Javaid

Copyright (C) 2020 Jiang Jie Sun et al. This is an open access article distributed under the Creative Commons Attribution License, which permits unrestricted use, distribution, and reproduction in any medium, provided the original work is properly cited.

Based on the situation of physician trust in the patient (PTP), we explored the differences in perceived behaviors of physician trust in the patient (PBPTP). We used the PTP scale as a research tool, taking physicians of the hospitals in Anhui region as the research object to carry out the investigation of PTP, Python software was applied to explore the status of PTP, and the differences of PBPTP distribution rate with different demographic characteristic variables were compared by testing based on theory of planned behavior. We get six results as follows: (1) the overall PTP level was low, and nearly $50 \%$ of doctors doubt the integrity of patients. "Patients will not be driven by improper interests" becomes the most reluctant problem or the most distrustful option for doctors. (2) In terms of patients' participation in disease management and regular follow-up visits, PTP rate in male was higher than that in female (Ps < 0.018). (3) PBPTP was affected by age (Ps < 0.017). (4) In terms of the behavior of patients who did not follow the treatment plans, the PTP rate of postgraduates and above physicians was higher than that of undergraduates and below $(P=0.017)$. (5) In terms of providing diagnosis and treatment information, timely notification of illness, medication information, doctor-patient communication behaviors, and compliance with doctors' treatment plans, PBPTP was affected by doctors' professional titles and annual income levels (Ps $\leq 0.001$ ), At the same time, PTP levels of different professional titles showed differences in patients' respect for doctors' time and bottom line $(\mathrm{Ps} \leq 0.001)$. (6) In terms of doctor-patient communication behaviors, PBPTP was affected by physician departments $(P \leq 0.001)$. Hence, demographic characteristics variable may be one of the factors affecting PBPTP, and PBPTP is associated with doctor-patient risk. It makes sense for us to propose a new model of physician-patient risk management from the perspective of PTP about "official-individual-social" triple action.

\section{Introduction}

The article "Cultivating the good root of doctor-patient trust" in the People's Daily pointed out that the current domestic doctor-patient trust is fragile; medical violence and injury to physicians occur frequently; the mutual trust and harmonious relationship between doctors and
patients(RBDP) that originally "health is related to life" has changed. How to enhance the trust between doctors and patients and avoid the risk that doctors and patients have become a research hotspot in academia. The outline of the "healthy China 2030" program emphasizes on strengthening humanistic care for medical services and building a harmonious RBDP. These articles proposed that the doctor- 
patient trust crisis is the important reason for the deterioration of RBDP, and the lack of doctor-patient trust is the root cause [1-3]. Through the questionnaire survey, it is found that the current doctor-patient trust status and doctor-patient trust level have an impact on RBDP. The results are as follows (Figure 1).

The results show that the overall level of doctor-patient trust is not high. Both doctors and patients highly believe that doctor-patient trust has a great impact on RBDP, while doctors pay more attention to doctor-patient trust. The early manifestation of the doctor-patient risk is the deterioration of the RBDP. Therefore, doctor-patient trust is a direct factor influencing doctor-patient risk. Krot conducted an online study about polish patients and concluded that doctor-patient trust positively affects RBDP [4].

The research on doctor-patient trust originated earlier, mainly including the research of doctor-patient trust connotation and doctor-patient trust measurement methods.

Doctor-patient trust is a belief, an expectation, a fulcrum, or a feeling of security (reflecting the emotional nature), etc. For example, Thom proposed that doctor-patient trust is the patient's expectation of the medical services provided by the doctor [5]; Haslam believed that trust is the fulcrum of RBDP [6]; Howe believed that doctor-patient trust means that the patient trusts the doctor [7]. This view dominated for a long time; however, the degree of doctors' trust in patients affects the perception of communication quality and is directly related to doctor-patient risk [8]. At present, there is almost no research on the status of doctor-patient trust and doctor-patient risk management. This paper explores the new idea of doctor-patient risk management from the perspective of doctor-patient trust, which has certain theoretical value.

Doctor-patient trust measurement research originated in 1973. Wallston introduced the "interpersonal trust scale" into the medical field and proposed the "nurse trust patient scale", which apparently solved the problem of nurse trust patient measurement [9]. Because of the lack of reliability and validity as well as the Cronbach coefficient of retest reliability is only 0.32 , the scale is considered invalid. In 1991, Anderson first proposed the scale of trusting doctors, which includes three dimensions, trustworthiness, confidence, and information, and 11 items. Using quantitative methods to measure the trust of patients in doctors has become one of the main reference standards for such studies since that [10]. In 2015, Gopichandran proposed the trust doctor scale from the five dimensions of the doctor's perception, treatment assurance, confidence in the doctor, loyalty to him/her, and respect for him/her and verified that the overall model had a good statistical fitting effect [11]. However, most of them lack integrity reliability and validity test [12-14]. Until 2016, Dong translated the physician trust patient scale developed by Thom and revised the Chinese version [15]. The application of measurement of doctorpatient trust appears in China.

As a contradiction between the supply of medical resources in China and the growing demand for medical services in patients have intensified, the risks of doctors and patients have gradually increased, and the tension between

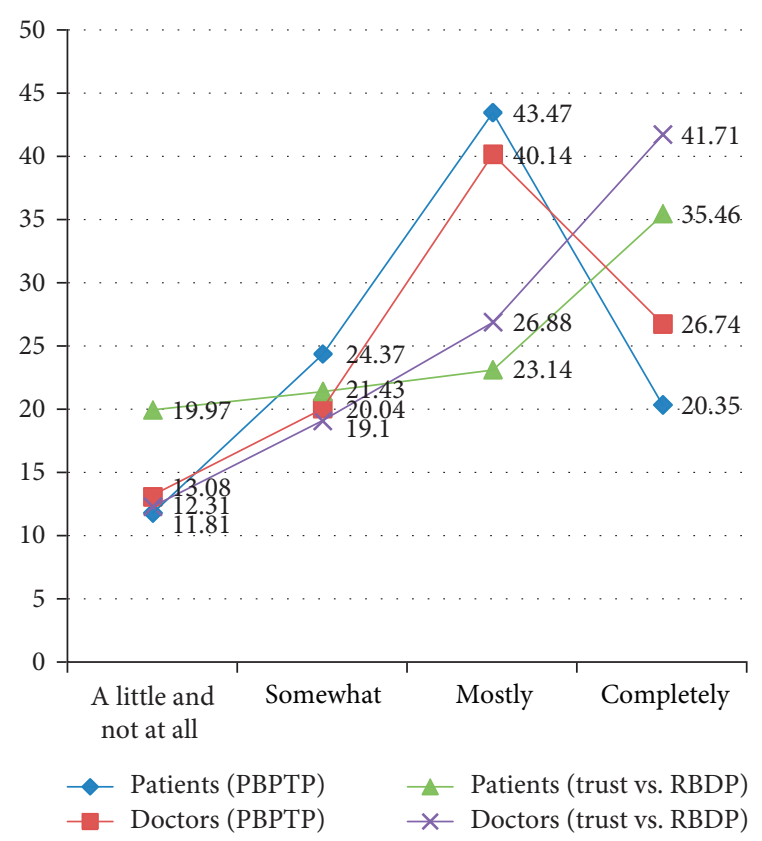

Figure 1: The results of PBPTP and trust level vs. RBDP (\%). Note. RBDP, relationship between doctors and patients.

doctors and patients has become a social hotspot. Trust between doctors and patients is the basis of building a harmonious RBDP as well as an important guarantee for obtaining the best medical effect [16]. The crisis of doctorpatient trust has become an urgent social problem in China while the study of doctor-patient risk in Anhui has certain representativeness in the country. Investigating and studying the current situation of physicians' trust in patients in Anhui hospitals is a key step in solving this core problem. The Theory of Planned Behavior holds that human behavior is the result of deliberate and planned thinking and that perceptual behavior, attitude, and subjective norms affect behavioral intention and contribute to the process of actual behavior [17]. Based on this, this study explores the status quo of cognitive of physician trust in the patient (PBPTP) and the representation of PBPTP in demographic variables and then explores a new doctor-patient risk management model based on doctor-patient Trust. Thom put forward the importance of PBPTP and pointed out that doctors' trust in patients can positively affect patients' trust in doctors, and the lack of trust in related doctors will lead patients to think that doctors' behaviors is lax and negative and may affect patients' behavior [18]. He proposed that the current RBDP is tense [19], and Sun interpreted the economic incentives for the frequent doctor-patient risks [20]. On this basis, we propose Hypothesis 1.

Hypothesis 1. The overall trust of physicians in trusting patients is not high; Sun proposed that physicians' cognitive level of RBDP evaluation is affected by factors such as age, education, job title, and income level [21]. Some studies have proposed that the risk of surgical doctors and patients is relatively high [22], and the conflict rate between doctors 
and patients may negatively affect the PTP rate. On this basis, we propose Hypothesis 2 .

Hypothesis 2. PBPTP differs in gender, age, job title, education level, and annual income level; Zhang's research shows that only $2.4 \%$ of physicians are satisfied with their working environment [23]. Doctors, who are dissatisfied with their working environments, are more likely to avoid high-risk diagnosis and treatment at work, whose attitude may further stimulate patients' dissatisfaction with doctors and affect the level of doctor-patient mutual trust. However, because of different departments, the working environment is relatively different, so we propose Hypothesis 3 here.

Hypothesis 3. There are department differences in PBPTP.

\section{Objects and Methods}

2.1. Research Samples. This paper took doctors from 10 hospitals in Anhui province as the objects of investigation and conducted a questionnaire survey on doctors from various departments including internal medicine, surgery, obstetrics and gynecology, and pediatrics (choose the opportunity of weekly meeting of corresponding hospital to conduct questionnaire survey). The research sample size is not less than 10 times of the number of observed variables to ensure the validity of the research results [24]. Therefore, the sample size selected in this study is 30 times more than the observed variables. A total of 350 copies were distributed and 329 valid questionnaires were collected, with an effective recovery rate of $94 \%$. The survey was conducted by postgraduate students of psychology, lecturer of psychology, and associate professor of psychology. Before the questionnaire was distributed, the respondents received a unified professional training and obtained the informed consent of the respondents.

\subsection{Research Tools}

2.2.1. Physician Trust in the Patient Scale (PTP Scale). This scale was developed by Thom et al. [5]. Until 2016, Dong translated the physician trust patient scale developed by Thom and revised the Chinese version [15]. In 2018, Sun used the doctor-patient trust scale to explore the relationship between doctor-patient trust and mental resilience of doctors and concluded that the higher degree of doctor-patient trust, the better doctor-patient relationship [25]. This scale is mainly used to measure the level of PBPTP, including patient roles (8 items in total) and respect for interpersonal relationships (4 items in total). Each item has 5 options from (no trust at all) to (full trust), measuring the levels of doctors' perception of patients' trust. The Cronbach coefficient of the scale is 0.92 , which has a good reliability. Each item of this scale reflects PBPTP, and the reliability and validity of each item is high, meeting the needs of this study.

2.2.2. Self-Compiled Demographic Data Questionnaire. Demographic information about the respondents (gender, age, education level, professional title, and income level) and hospital departments were included (internists, surgeon, gynecologist, pediatrics, and other clinical departments).

2.2.3. Statistical Methods. After all questionnaires were reviewed by two graduate students, EpiData3.1 was used to input data, python software was used to explore the status quo of PTP, SPSS19.0 software was used for analysis [26], frequency and percentage of counting data were used for statistical description, and chi-squared test was used to compare the differences of PBPTP among different demographic characteristics. $P<0.05$ was considered statistically significant.

\section{Results}

3.1. Descriptive Statistics. This study collected data from 329 doctors, including 162 males (49.24\%) and $167 \mathrm{fe}$ males $(50.76 \%)$. This sample was predominantly doctors from graduate/professional school(69.60\%), but there were also college graduate $(26.44 \%)$ and junior college doctors $(3.96 \%) .95$ people $(28.88 \%)$ have primary professional titles, 81 people $(24.62 \%)$ have intermediate professional titles, 105 people (31.91\%) have sub-senior professional titles, and 48 people (14.59\%) have senior professional titles. There were 72 patients $(21.88 \%)$ in internal medicine, 72 patients $(21.88 \%)$ in surgery, 62 patients $(18.84 \%)$ in gynecology, 64 patients $(19.45 \%)$ in pediatrics, and 59 patients (17.95\%) in other departments. There were 90 persons $(27.36 \%)$ with an annual income of less than $¥ 70,000,104$ persons $(31.61 \%)$ with an annual income of between $¥ 70,000$ and $¥ 120,000$, and 135 persons (41.03\%) with an annual income of 120,000 (¥) and above; average age was 37.8 , standard deviation was 11.2 , with the youngest being 23 and the oldest 61 .

\subsection{Distribution of Perceived Behaviors of Doctors Trusting} Patients. This study uses Python-2.7.15 software to analyze the PBPTP data in a heat map, and the results show that the overall trust of each PBPTP project is not high (See Figure 2 for details).

Physicians strongly believe that the patient's perceived behavior is "the patient is willing to inform you of important changes in his/her condition in a timely manner", accounting for only $54.1 \%$. The perception behavior with the highest complete trust rate is "the patient will provide all the medical information you need", accounting for only $21.6 \%$. On the contrary, the perceived behavior with the highest total distrust rate is "patients will not be driven to seek treatment by improper benefits", as high as $44.7 \%$. Nearly half of the participants questioned the patient's integrity issues, which became the most unwilling physician or the least trusted option. Combined with PBPTP heat map, Hypothesis 1 is confirmed.

Next, we will use the chi-square test of $R \times C$ cross-table data to explore the PBPTP distribution rates of different demographic characteristics. Its statistics is: 


\begin{tabular}{|c|c|c|c|c|}
\hline 1.2 & 6.7 & 21.6 & 48.9 & 21.6 \\
\hline 1.2 & 6.4 & 17.9 & 54.1 & 20.4 \\
\hline 3 & 6.1 & 26.8 & 46.8 & 17.3 \\
\hline 1.2 & 4.9 & 21.6 & 52.9 & 19.5 \\
\hline 2.1 & 12.8 & 34.7 & 42.9 & 7.6 \\
\hline 4.6 & 20.7 & 42.9 & 25.8 & 6.1 \\
\hline 17.9 & 31.6 & 27.1 & 18.2 & 5.2 \\
\hline 10 & 31.6 & 31.6 & 21.3 & 5.5 \\
\hline 18.2 & 34.3 & 24 & 17.6 & 5.8 \\
\hline 17.3 & 35 & 27.4 & 15.5 & 4.9 \\
\hline 28.3 & 35.9 & 23.4 & 10.3 & 2.1 \\
\hline 44.7 & 23.4 & 19.8 & 9.1 & 3 \\
\hline 0 & 1 & 2 & 3 & 4 \\
\hline
\end{tabular}

FIGURE 2: Heat map of the distribution of physician's trust rate in patients' perception behavior (\%). Note. (a) the patient will provide all the medical information you need; (b) the patient is willing to inform you of important changes in his/her condition in a timely manner; (c) the patient will tell you all the drugs he/she is taking or the treatment he/she is receiving; (d) the patient can understand what you say to him/her; (e) the patient will follow the treatment plan you suggest; (f) the patient will actively participate in his/her condition management; ( $\mathrm{g}$ ) the patient will tell you when they do not follow the treatment plan; (h) the patient will have regular follow-up visits; (i) the patient will respect your time; (j) the patient will respect your personal bottom line; $(\mathrm{k})$ patient will not make unreasonable demands; (l) patient will not be driven by improper interests to see a doctor; 0 , no trust at all; 1 , a little trust; 2 , a certain trust; 3 , a great trust; 4 , full trust.

$$
\chi^{2}=\sum_{i=1}^{R} \sum_{j=1}^{C} \frac{\left(A_{i j}-T_{i j}\right)^{2}}{T_{i j}}
$$

where $A_{i j}$ is the actual frequency of each cell, $T_{i j}$ is the theoretical frequency of each cell, and its parametric freedom is calculated as $(R-1)(C-1), R$ is the number of rows, and $C$ is the number of columns.

There are differences in perceived behaviors of PTP with different demographic characteristics.

We performed the chi-squared test on PBPTP distribution rates of different genders/ages. "Patient will actively participate in his/her disease management" and "patients will visit regularly" male physicians have a higher PTP rate than female physicians (all $P<0.018$ ), and there is no significant difference in other items. The age difference of PBPTP distribution rate was statistically significant (all $P<0.017$ ), which partially verified Hypothesis 2. Detailed data are shown in Table 1.

We performed a chi-squared test on the PBPTP distribution rate for different levels of education/title. In terms of perceived behaviors of "patients will tell you when they do not follow the treatment plan", the PTP rate of postgraduates and above is higher than undergraduates and below $(P=0.017)$, and there is no significant difference in other programs. In addition to the items "patient will actively participate in his/her condition management", "patient will tell you when he does not follow the treatment plan", "patient will return regularly," and "patient will not make unreasonable requests" with the exception of "patients are not driven by improper benefits," the remaining perceived behaviors are statistically significant for different job title levels (Ps $\leq 0.001$ ), partially verifying Hypothesis 2, and the detailed data are shown in Table 2.

We conducted the chi-squared test on the trust rate of patients' perceived behaviors among physicians with different annual income/department. In the project "patients will provide you with all of the medical information you need", "patient inform you of the important changes about his/her illness timely", "patient will tell you the kinds of drugs he/she is taking or the treatment he/she is accepting", "the patient can understand what you said to him/her," and "patients will follow the treatment plans proposed by you", the perceived behaviors and PBPTP distribution income are different $(P \leq 0.001)$. The difference in other items is not obvious, which partially confirms the conjecture of Hypothesis 2; in terms of "the patient can understand what you say to him/her," the PBPTP distribution rate was different among departments $(P \leq 0.001)$. The conjecture of $\mathrm{Hy}-$ pothesis 3 is partially confirmed, and the detailed data are shown in Table 3.

\section{Discussion}

This study found that the overall level of PTP was not high, and most PBPTPs were skeptical. There are three possible reasons for this. Firstly, it will take some time for the medicine to take effect, and in such a semistructured medical market environment [27], it is very common for patients to change therapists because they cannot adhere to the medication, which aggravates the suspicion between doctors and patients. Secondly, it may be related to the current domestic doctor-patient tension, frequent doctor-patient disputes, frequent occurrence of medical reparations, and severe impact on PBPTP caused by medical trouble and medical violence, which is consistent with the literature $[19,21]$. Furthermore, it may be related to domestic medical habits. For example, in the face of major medical activities such as the diagnosis and treatment plan and operation, doctorpatient communication and medical respect are mostly process-based and off-site behaviors, which have adversely affected the patients/families. As a result, the patients'/ family's bad emotions will be directly fed back to the physician, which will affect PBPTP cognition, so the overall low PBPTP level can be understood. Based on such issues, we propose strengthening the top-level design and official intervention, reducing the imbalance of doctor-patient information, and improving doctor-patient cognition.

The results of this study showed that the distribution rate of PBPTP was correlated with factors such as gender, age, education level, professional title, and department of physicians, which was consistent with the results of the literature $[14,25]$. The reason for the association with gender may be the gender differences in personality traits and the consequences of gender differences in China over the last century. One of the reasons associated with age may be that the formation of individual thinking and values is influenced by 
TABLE 1: Comparison of gender/age differences between physicians and patients' perceived behavior trust (cases (\%)).

\begin{tabular}{|c|c|c|c|c|c|c|c|c|c|c|}
\hline \multirow{2}{*}{ Projects } & \multicolumn{2}{|c|}{ PBPTP $n(\%)$} & \multirow{2}{*}{$x^{2}$} & \multirow{2}{*}{$P$} & \multicolumn{4}{|c|}{ PBPTP $n(\%)$} & \multirow{2}{*}{$\chi^{2}$} & \multirow{2}{*}{$P$} \\
\hline & Male & Female & & & $21 \sim 30$ & $31 \sim 40$ & $41 \sim 50$ & $>51$ & & \\
\hline $\mathrm{a}$ & $114(70.4)$ & $118(70.6)$ & 0.003 & 0.997 & $36(40.9)$ & $72(66.7)$ & $82(96.5)$ & $42(87.5)$ & 72.073 & 0.000 \\
\hline $\mathrm{b}$ & $119(73.3)$ & $126(75.5)$ & 0.172 & 0.706 & $39(44.3)$ & $82(75.9)$ & $82(96.5)$ & $42(87.5)$ & 68.124 & 0.000 \\
\hline c & $109(67.3)$ & $102(61.1)$ & 1.377 & 0.252 & $40(45.5)$ & $58(53.7)$ & $77(90.6)$ & $36(75.0)$ & 46.781 & 0.000 \\
\hline $\mathrm{d}$ & $117(72.2)$ & $121(72.5)$ & 0.002 & 0.962 & $43(48.9)$ & $71(65.7)$ & $79(92.9)$ & $45(93.8)$ & 55.615 & 0.000 \\
\hline $\mathrm{e}$ & $83(51.2)$ & $83(49.7)$ & 0.077 & 0.781 & $28(31.8)$ & $28(25.9)$ & $73(85.9)$ & $37(77.1)$ & 94.514 & 0.000 \\
\hline$f$ & $64(39.5)$ & $41(24.6)$ & 8.464 & 0.004 & $25(28.4)$ & $25(23.1)$ & $41(48.2)$ & $14(29.2)$ & 14.904 & 0.002 \\
\hline g & $45(27.8)$ & $32(19.2)$ & 3.405 & 0.069 & $14(15.9)$ & $18(16.7)$ & $32(37.6)$ & $13(27.1)$ & 15.474 & 0.001 \\
\hline $\mathrm{h}$ & $53(32.7)$ & $35(31.0)$ & 5.802 & 0.018 & $15(17.0)$ & $22(20.4)$ & $34(40.0)$ & $17(35.4)$ & 15.930 & 0.001 \\
\hline $\mathrm{i}$ & $45(27.8)$ & $32(19.2)$ & 3.405 & 0.069 & $12(13.6)$ & $10(9.3)$ & $34(40.0)$ & $21(43.8)$ & 40.881 & 0.000 \\
\hline j & $40(24.7)$ & $27(16.2)$ & 3.684 & 0.057 & $12(13.6)$ & $8(7.4)$ & $27(31.8)$ & $20(41.7)$ & 33.879 & 0.000 \\
\hline $\mathrm{k}$ & $26(16.1)$ & $15(9.0)$ & 3.765 & 0.066 & $8(9.1)$ & $7(6.5)$ & $15(17.6)$ & $11(22.9)$ & 11.362 & 0.010 \\
\hline 1 & $24(14.8)$ & $16(9.6)$ & 2.109 & 0.177 & $9(10.2)$ & $6(5.6)$ & $16(18.8)$ & $9(18.8)$ & 10.205 & 0.017 \\
\hline
\end{tabular}

Note. The connotation of the project serial number is the same as that in Figure 3. Significance level $P<0.05$ (2-tailed). Referring to the literature [4], doctors with "great trust" and "complete trust" are denoted as patients trusted by doctors.

TABLE 2: Comparison of differences in trust in patients' perceived behavior among physicians with different education levels/professional titles (cases (\%)).

\begin{tabular}{|c|c|c|c|c|c|c|c|c|c|c|}
\hline \multirow{2}{*}{ Projects } & \multicolumn{2}{|c|}{ CPTP $n(\%)$} & \multirow{2}{*}{$\chi^{2}$} & \multirow{2}{*}{$P$} & \multicolumn{4}{|c|}{ CPTP n(\%) } & \multirow{2}{*}{$\chi^{2}$} & \multirow{2}{*}{$P$} \\
\hline & UGB & PGA & & & Primary & Intermediate & Subsenior & Senior & & \\
\hline $\mathrm{a}$ & $72(72.0)$ & $160(69.9)$ & 0.152 & 0.793 & $42(44.2)$ & $52(64.2)$ & $97(92.4)$ & $41(85.5)$ & 62.539 & 0.000 \\
\hline $\mathrm{b}$ & $78(78.0)$ & $167(73.0)$ & 0.943 & 0.410 & $46(48.4)$ & $58(71.6)$ & $100(95.2)$ & $41(85.5)$ & 61.098 & 0.000 \\
\hline c & $63(63.0)$ & $148(64.7)$ & 0.080 & 0.803 & $47(49.5)$ & $42(51.9)$ & $82(78.1)$ & $40(83.3)$ & 30.778 & 0.000 \\
\hline $\mathrm{d}$ & $76(76.0)$ & $162(70.7)$ & 0.962 & 0.351 & $45(47.4)$ & $58(71.6)$ & $90(85.7)$ & 45 (93.7) & 50.011 & 0.000 \\
\hline e & $55(55.0)$ & $111(48.5)$ & 1.187 & 0.284 & $30(31.6)$ & 34 (41.9) & $64(60.9)$ & $38(79.2)$ & 36.328 & 0.000 \\
\hline $\mathrm{f}$ & $27(27.0)$ & $78(34.1)$ & 1.597 & 0.247 & $29(30.6)$ & $23(28.4)$ & $37(35.2)$ & $16(33.3)$ & 1.124 & 0.771 \\
\hline g & $15(15.0)$ & $62(27.1)$ & 5.661 & 0.017 & 17 (17.9) & $17(21.0)$ & $29(27.6)$ & $14(29.1)$ & 3.802 & 0.284 \\
\hline h & $25(25.0)$ & $63(27.5)$ & 0.224 & 0.686 & $17(17.9)$ & $22(27.2)$ & $34(32.4)$ & $15(31.2)$ & 6.004 & 0.111 \\
\hline $\mathrm{i}$ & $19(19.0)$ & $58(25.3)$ & 1.555 & 0.258 & $10(10.5)$ & $14(17.3)$ & $35(33.4)$ & $18(37.5)$ & 21.576 & 0.000 \\
\hline j & $16(16.0)$ & $51(22.3)$ & 1.688 & 0.234 & $11(11.6)$ & $10(12.4)$ & $28(26.7)$ & $18(37.5)$ & 18.995 & 0.000 \\
\hline $\mathrm{k}$ & $13(13.0)$ & $28(12.2)$ & 0.038 & 0.857 & $7(7.4)$ & $7(8.7)$ & $18(17.1)$ & $9(18.8)$ & 7.191 & 0.066 \\
\hline 1 & $11(11.0)$ & $29(12.7)$ & 0.180 & 0.718 & $9(9.5)$ & $8(9.9)$ & $15(14.3)$ & $8(16.7)$ & 2.394 & 0.495 \\
\hline
\end{tabular}

Note. The connotation of the item serial number is the same as that in Figure 3; the significance level is $P<0.05$ (2-tailed). With reference to the literature [17], doctors with "great trust" and "full trust" are recorded as doctors trusting patients; UGB: undergraduates and below; PGA: postgraduates and above.

TABLE 3: Comparison of differences in patients' perceived behavior trust among physicians of different annual incomes/departments (cases $(\%))$.

\begin{tabular}{|c|c|c|c|c|c|c|c|c|c|c|c|c|}
\hline \multirow{2}{*}{ Projects } & \multicolumn{3}{|c|}{ СРТP $n(\%)$} & \multirow{2}{*}{$\chi^{2}$} & \multirow{2}{*}{$P$} & \multicolumn{5}{|c|}{ CPTP $n(\%)$} & \multirow{2}{*}{$\chi^{2}$} & \multirow{2}{*}{$P$} \\
\hline & $<70000$ & $70000 \sim 120000$ & $>120000$ & & & Internists & Surgeon & Gynecologist & Pediatrics & Others & & \\
\hline $\mathrm{a}$ & $45(50.0)$ & $63(60.6)$ & $124(91.8)$ & 52.721 & 0.000 & $55(76.3)$ & $47(65.3)$ & $51(82.3)$ & $43(67.2)$ & $36(61.0)$ & 9.158 & 0.057 \\
\hline $\mathrm{b}$ & $51(56.6)$ & $68(65.4)$ & $126(93.4)$ & 44.783 & 0.000 & 59 (81.9) & $53(73.6)$ & $47(75.8)$ & $50(78.1)$ & $36(61.0)$ & 8.268 & 0.082 \\
\hline c & $49(54.4)$ & $53(51.0)$ & $109(80.7)$ & 27.704 & 0.000 & $54(75.0)$ & $39(54.2)$ & $40(64.5)$ & $43(67.2)$ & $35(59.3)$ & 7.663 & 0.105 \\
\hline $\mathrm{d}$ & $45(50.0)$ & $75(72.1)$ & $118(87.4)$ & 37.768 & 0.000 & $45(62.5)$ & $40(55.5)$ & $51(82.3)$ & $55(85.9)$ & $47(79.6)$ & 24.164 & 0.000 \\
\hline e & $28(31.1)$ & $49(47.2)$ & $89(65.9)$ & 26.863 & 0.000 & $32(44.5)$ & $38(52.8)$ & $33(53.2)$ & $37(57.8)$ & $26(44.1)$ & 2.891 & 0.576 \\
\hline$f$ & $31(34.4)$ & $31(29.8)$ & $43(31.9)$ & 0.478 & 0.787 & $18(25.0)$ & $29(40.3)$ & $15(24.2)$ & $25(39.1)$ & $18(30.5)$ & 7.161 & 0.128 \\
\hline g & $16(17.8)$ & $26(25.0)$ & 35 (25.9) & 2.216 & 0.330 & $16(22.2)$ & $22(30.5)$ & $10(16.1)$ & $14(21.9)$ & $15(25.4)$ & 4.158 & 0.385 \\
\hline h & $20(22.2)$ & $30(28.8)$ & $38(28.1)$ & 1.310 & 0.520 & $17(23.6)$ & $22(30.5)$ & $18(29.1)$ & $18(28.1)$ & $13(22.0)$ & 1.791 & 0.774 \\
\hline i & $13(14.4)$ & $29(27.9)$ & $35(25.9)$ & 5.674 & 0.059 & $16(22.2)$ & $15(20.8)$ & $15(24.2)$ & $14(21.9)$ & $17(28.8)$ & 1.390 & 0.846 \\
\hline j & $12(13.3)$ & $20(19.2)$ & $35(25.9)$ & 5.401 & 0.067 & $13(18.1)$ & $14(19.4)$ & $12(19.4)$ & $8(12.5)$ & $20(33.9)$ & 9.418 & 0.051 \\
\hline $\mathrm{k}$ & $8(8.9)$ & $13(12.5)$ & $20(14.9)$ & 1.738 & 0.419 & $5(7.0)$ & $13(18.1)$ & $8(12.9)$ & $9(14.0)$ & $6(10.2)$ & 4.520 & 0.340 \\
\hline 1 & $12(13.3)$ & $10(9.7)$ & $18(13.3)$ & 0.921 & 0.631 & $7(9.7)$ & $9(12.5)$ & $5(8.1)$ & $11(17.2)$ & $8(13.6)$ & 3.005 & 0.557 \\
\hline
\end{tabular}

Note. The connotation of the project serial number is the same as that in Figure 3. Significance level $P<0.05$ (2-tailed). Referring to [17], doctors with "great trust" and "complete trust" are denoted as patients trusted by doctors. Others: other clinical departments.

age, and the other may come from the formation of individual cognition and the differences in the influence of social environment and doctor-patient risk events. The possible reason for the influence of education level and professional title is the management system and promotion mechanism of domestic health service. Differences among departments 
may result from different incidence rates of doctor-patient risk events in departments, which is consistent with the literature [11]. Based on such problems, we propose to actively respond to personalized needs, analyze personalized differences, weigh the pros and cons, improve the advantages of policy management, eliminate the disadvantages of the old pattern, improve the medical effect, and reduce the doctor-patient risk from the root.

The results of this study found that the trust rate of doctors in patients' perceived behaviors fluctuated around $20 \%$ for five items, such as "not following the advice of treatment plan", "patients will respect your time", "patients will respect your personal bottom line", "patients will not make unreasonable demands," and "patients will not be driven by illegitimate interests". In particular, "patients will not make unreasonable demands" and "patients will not be driven by illegitimate interests" became the most reluctant problems for doctors to face or the least trusted option. There are several possible reasons for this. Firstly, unfair media coverage of doctor-patient disputes has seriously affected doctors' personal cognition. Secondly, hospitals and even the officials regard the affected party as vulnerable groups; nonprinciple compromises and financial compensation have made the doctor be swayed by considerations of gain and loss of consciousness. Thirdly, the absence of relevant regulations and laws leads to the lack of rules and regulations of patients/family members in doctor-patient disputes, which seriously affects the basic image of patients, thus giving PBPTP a bias. Based on such issues, we propose to enhance the effect of third-party supervision, increase social participation, and face up to the risks of doctorpatient supply and demand.

\section{Conclusion}

Through the above analysis and research, in order to improve RBDP, enhance doctor-patient trust rate, and promote RBDP harmony, doctor-patient risk management models of "Official-Individual-Social" Triple Action should be formed, as shown in Figure 3.

Official actions, first of all, further standardize the development of medical and health services, strengthen the reform of medical and health care, implement scientific pricing of pharmaceutical products, simplify the distribution process, save health resources, and form a good situation of "the people can afford to be ill and is optimistic about the disease". Secondly, improve the training mechanism for medical workers, optimize the mechanism for staff promotion, and explore a "green" salary system that links doctors' cure rates, patient satisfaction rates, and bonuses; finally, improve the legal system for doctor-patient disputes, regulate doctor-patient rights and responsibilities, and establish dispute adjustment mechanism.

Individual response clarified the consequences of medical atrocity and clarified the significance of doctorpatient mutual trust. First of all, to explore the relationship between "doctor-patient trust" and "social trust" by exploring the validity of doctor-patient trust

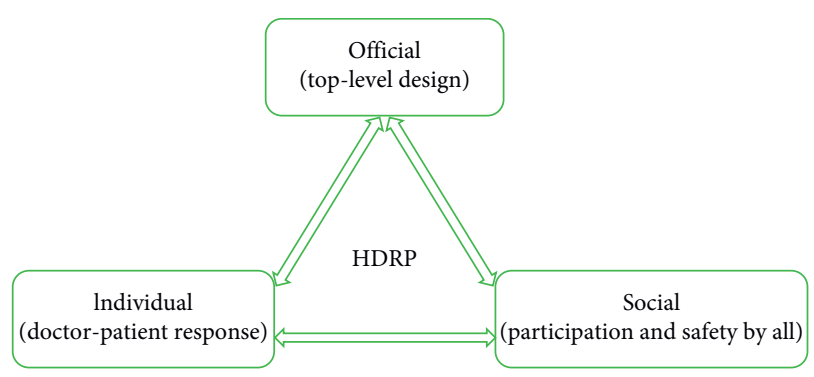

Figure 3: Model of "official-individual-social" triple action. Note. HDPR: harmonious RBDP.

and behavioral intervention and to open up a theoretical framework to improve individual trust rate from an academic perspective is an attempt to solve the crisis of social trust and doctor-patient trust; secondly, carry out the publicity of laws and regulations on doctor-patient disputes, so that patients/family members can clearly understand the serious consequences of medical violence, smooth the channels of doctor-patient dispute mediation, and release the extreme emotions of patients/ family members; Finally, the concept of medical personnel should be changed, the professional spirit of medical personnel should be improved, and the passive should be changed into the active one, so as to form a medical team that is "dare to treat and able to treat" diseases.

The social supervision medical process gradually realizes "full openness". Firstly, promote the popularization of medical science to the general public, try to make the whole people agree with the correct medical treatment concepts such as "treatment is not equal to cure" and "doctors do not cure all diseases," so as to guide patients/families to have reasonable expectations on the treatment effect and reduce conflicts. Secondly, social media should be impartial, scientific, and reasonable in shouldering corresponding social responsibilities, be impartial, rational, and tolerant in reporting medical disputes, and have clear rights and responsibilities, while analyzing doctor-patient contradictions fairly, so as to make the due contribution to construct the good health care environment.

To summarize, demographic characteristics variable may be one of the factors affecting PBPTP; PBPTP is associated with doctor-patient risk. It makes sense for us to propose a new model of physician-patient risk management from the perspective of PTP about "official-individual-social" triple action.

This study expands the research on the relationship between PBPTP and demographic characteristics, provides a new research perspective for RBDP improvement and doctor-patient risk management in hospitals, and expands the application of planned behavior theory in doctor-patient risk management in hospitals, which makes certain theoretical contributions. Its deficiency is that it has not conducted quantitative management of doctor-patient risk management based on differences in PBPTP level, which is also an in-depth study that we will carry out in the future. 


\section{Data Availability}

All datasets generated for this study are included in the article and/or the supplementary files.

\section{Ethical Approval}

Ethical approval for the study was received from the Ethics Committee of Anhui Medical University, and this study meets the ethical requirements.

\section{Disclosure}

The funders had no role in study design, data collection and analysis, decision to publish, or preparation of the manuscript.

\section{Conflicts of Interest}

The authors declare that they have no conflicts of interest.

\section{Authors' Contributions}

Jiang Jie Sun was involved in the study design, data-entry, and original writing. Zhi Bo Zheng was involved in the study design. Xue Li Jiang was responsible for the data entry and language editing. Wei Wei $\mathrm{Hu}$ was responsible for data entry. Nan Zhen Ma and Xiao-Zhe Yan collected the data. Jun Liu was responsible for language editing. Meng Ying Li was involved in the data analysis and language editing. Cheng sen He financially supported the study. Li ping Zhang was involved in the language editing, data collection, and financial support.

\section{Acknowledgments}

We would like to thank Matthias Alfons Theis for collecting part of the data. This work was supported in part by the Natural Science Foundation of Anhui Province of China under Grant no. 1908085MG233 to JS, Quality Engineering for research projects of the Anhui Department of Education about Wisdom classroom 2018zhkt180 to JS, the Natural Science Foundation for the Higher Education Institutions of Anhui Province of China under Grant no. KJ2019A0945 to LZ, and Science and Technology Innovative Research Team in Higher Educational Institutions of Hunan Province under Grant nos. KJ2016A372 and KJ2016SD30 to LZ and CH. The work was supported in part by the Joint Special Foundation on basic research in Local Colleges and Universities for the Department of Science and Technology of Yunnan Province of China under Grant no. 2017FH001-106 to ZZ.

\section{References}

[1] C. Zhou and X. Xu, "Review on the studies of doctor-patient trust in both China and overseas countries," Journal of Kunming University of Science and Technology(Social Science Edition), vol. 15, no. 1, pp. 8-14, 2015.

[2] J. Liu, C. Zhou, and F. Yang, "A review on domestic and oversea literature about doctor-patient trust scale research,"
Journal of Kunming University of Science and Technology (Social Science Edition), vol. 15, no. 3, pp. 11-18, 2015.

[3] A. A. Sewell, "Disaggregating ethnoracial disparities in physician trust," Social Science Research, vol. 54, no. 11, pp. 1-20, 2015.

[4] W. Augustyniak, "The role of trust in doctor-patient relationship: qualitative evaluation of online feedback from polish patients," Economics \& Sociology, vol. 9, no. 3, pp. 76-88, 2015.

[5] D. H. Thom, M. A. Hall, and L. G. Pawlson, "Measuring patients' trust in physicians when assessing quality of care," Health Affairs, vol. 23, no. 4, pp. 124-132, 2004.

[6] D. Haslam, "Trust is the fulcrum of the doctor-patient relationship," The Practitioner, vol. 259, no. 1783, p. 35, 2015.

[7] E. G. Howe, "How to retain the trust of patients and families when we will not provide the treatment they want," The Journal of Clinical Ethics, vol. 26, no. 2, pp. 89-99, 2015.

[8] H. Pin, H. Jin, J. Eshin et al., "Socially transmitted placebo effects," Nature Human Behavior, vol. 3, pp. 1295-1305, 2019.

[9] K. A. Wallston, B. S. Wallston, and S. Gore, "Development of a scale to measure nurses' trust of patients: a preliminary report,” Nursing Research, vol. 22, no. 3, pp. 232-235, 1973.

[10] L. A. Anderson and R. F. Dedrick, "Development of the trust in physician scale a measure to assess interpersonal trust patient-physician relationships," Psychological Reports, vol. 67, no. 3, pp. 1091-1100, 1991.

[11] V. Gopichandran and S. K. Chetlapalli, "Trust in the physician-patient relationship in developing healthcare settings: a quantitative exploration," Indian Journal of Medical Ethics, vol. 12, no. 3, pp. 141-148, 2015.

[12] D. J. Burgess, "Addressing racial healthcare disparities: how can we shift the focus from patients to providers?" Journal of General Internal Medicine, vol. 26, no. 8, pp. 828-830, 2011.

[13] E. M. A. Smets, M. A. Hillen, K. F. L. Douma, L. J. A. Stalpers, C. C. E. Koning, and H. C. J. M. de Haes, "Does being informed and feeling informed affect patients' trust in their radiation oncologist?" Patient Education and Counseling, vol. 90, no. 3, pp. 330-337, 2013.

[14] S. Ozawa and P. Sripad, "How do you measure trust in the health system? a systematic review of the literature," Social Science \& Medicine, vol. 91, no. 1, pp. 10-14, 2013.

[15] Z. Dong and C. Chen, "A preliminary study of validity and reliability of the Chinese version of the Physician Trust in the Patient Scale," Chinese Mental Health Journal, vol. 30, no. 7, pp. 481-485, 2016.

[16] A. Banerjee and D. Sanyal, "Dynamics of doctor-patient relationship: a cross-sectional study on concordance, trust, and patient enablement," Journal of Family and Community Medicine, vol. 19, no. 1, pp. 12-19, 2012.

[17] I. Ajzen and M. Fishbein, Understanding Attitudes and Predicting Social Behavior, Prentice-Hall, Upper Saddle River, NJ, USA, 1980.

[18] D. H. Thom, S. T. Wong, D. Guzman et al., "Physician trust in the patient: development and validation of a new measure," The Annals of Family Medicine, vol. 9, no. 2, pp. 148-154, 2011.

[19] C. He, "Inspiration of the evolution of doctor-patient relationship to today's medical care reform," Jianghuai Tribune, no. 2, pp. 117-121, 2015.

[20] J. Sun, L. Zhang, C. He et al., "Interpretation of the Behavioral economics and countermeasures of the Medical risk," Journal of Nanjing Medical University(Social Sciences), vol. 73, no. 2, pp. 141-145, 2016.

[21] J. J. Sun, L. P. Zhang, P. K. Mu et al., "Differences in the cognitive evaluation of the doctor-patient Relationship 
between the medical side and the contracting parties," Chinese Mental Health Journal, vol. 30, no. 7, pp. 486-491, 2016.

[22] J. Sun, L. Zhang, and C. He, "The related factors of physician trust in patients," Chinese Mental Health Journal, vol. 32, no. 5, pp. 407-409, 2018.

[23] X. Zhang and M. Sleeboom-Faulkner, "Tensions between medical professionals and patients in mainland China," Cambridge Quarterly of Healthcare Ethics, vol. 20, no. 3, pp. 458-465, 2011.

[24] J. Sun, R. Sun, Y. Jiang et al., "The relationship between psychological health and social support: evidence from physicians in China," PLoS One, vol. 15, no. 1, Article ID e0228152, 2020.

[25] J. Sun, L. Zhang, R. Sun et al., "Exploring the influence of resiliency on physician trust in patients: an empirical study of Chinese incidents," PLoS One, vol. 13, no. 12, Article ID e0207394, 2018.

[26] J. Sun, P. Wang, Y. Du, and J. Liu, "Analysing the influence factors of single task pricing based on public packet system: an Empirical Study in China," Journal of Physics: Conference Series, vol. 1437, pp. 1-6, 2020.

[27] Q. Yang and J. Pan, "Control under times of uncertainty: the relationship between hospital competition and physicianpatient disputes," International Journal for Equity in Health, vol. 16, no. 1, pp. 205-217, 2017. 